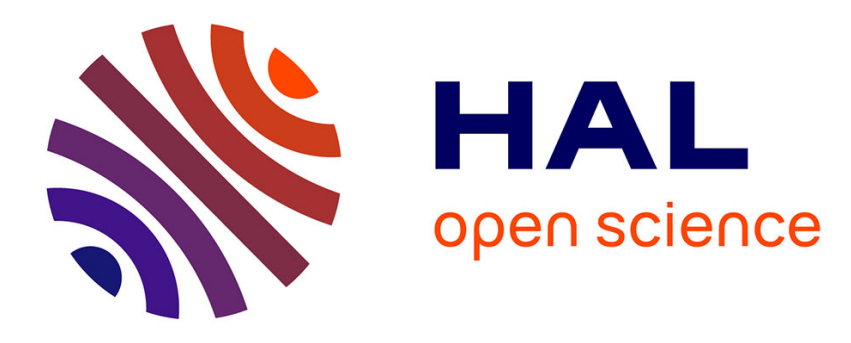

\title{
Cauchy Matrices in the Observation of Diffusion Equations
}

Faker Ben Belgacem, Sidi-Mahmoud Kaber

\section{To cite this version:}

Faker Ben Belgacem, Sidi-Mahmoud Kaber. Cauchy Matrices in the Observation of Diffusion Equations. journal of mathematical studies, 2015, 48 (4), pp.330-344. hal-01026457

\section{HAL Id: hal-01026457 https://hal.sorbonne-universite.fr/hal-01026457}

Submitted on 22 Jul 2014

HAL is a multi-disciplinary open access archive for the deposit and dissemination of scientific research documents, whether they are published or not. The documents may come from teaching and research institutions in France or abroad, or from public or private research centers.
L'archive ouverte pluridisciplinaire HAL, est destinée au dépôt et à la diffusion de documents scientifiques de niveau recherche, publiés ou non, émanant des établissements d'enseignement et de recherche français ou étrangers, des laboratoires publics ou privés. 


\title{
Cauchy Matrices in the Observation of Diffusion Equations
}

\author{
Faker Ben Belgacem* Sidi Mahmoud Kaber $^{\dagger}$
}

May 22, 2014

\begin{abstract}
Observability Gramians of diffusion equations have been recently connected to infinite Pick and Cauchy matrices. In fact, inverse or observability inequalities can be obtained after estimating the extreme eigenvalues of these structured matrices, with respect to the diffusion semi-group matrix. The purpose is hence to conduct a spectral study of a subclass of symmetric Cauchy matrices and present an algebraic way to show the desired observability results. We revisit observability inequalities for three different observation problems of the diffusion equation and show how they can be (re)stated through simple proofs.
\end{abstract}

KEYWORDS: Pick matrices, Cauchy matrices, Hadamard product, diffusion equation, observability inequalities.

\section{Introduction}

Observability inequalities are the milestone for the HUM method applied to nullcontrollability problems (see [20,21]). These inequalities are hard to prove in particular for infinite dimensional parabolic problems. A lot of work have been done for the observability of the heat equation. Consult the non-exhaustive list

\footnotetext{
*Sorbonne Universités, UTC, EA 2222, Laboratoire de Mathématique Appliquée de Compiègne, F-60205 Compiègne

†Sorbonne Universités, UPMC Univ Paris 06, UMR 7598, Laboratoire Jacques-Louis Lions, F-75005, Paris
} 
$[10,27,12,8, ?, 17,18,11,24,26,19]$. We are, of course, far from supplying a state-of-the-art on the subject.

We revisit some of the observability estimates in the diffusion problems in one dimension and give alternative proofs using algebraic tools such as spectral estimates of some structured matrices. Connection between the controllability of parabolic problems and Cauchy and Pick matrices has been pointed out in [1]. This remark allowed to state the severe ill-posedness degree of the exact controllability of the heat equation. A deeper analysis of these matrices results in algebraic proofs of the observability inequalities. The central element in the analysis is the closed form of the inverse of Cauchy matrices (see [6]). The first observation problem we study is the one analyzed in [8]. The second one is a boundary observation problem investigated in [24, 1]. The third and last one is the distributed observability (see, eg, [36]). Notice that for this last problem, the spectral analysis of the structured matrices needs to be complemented by Schur's theorem to provide bounds of the eigenvalues of the Hadamard entrywise product of matrices.

An outline of the paper is as follows. In Section 2, we investigate spectral properties of a countably infinite Pick matrix with respect to the diffusion semi-group matrix. The spectral equivalence between Pick matrix and symmetric Cauchy matrix is stated in the preliminary step. Then, we take profit of the closed form of the inverse of Cauchy matrices to state the desired comparison result. In Section 3, three observation problems are revisited. We follow the methodology by Fattorini and Russel [10]. However, instead of considering a moment equation as in $[8,12,36,34]$, we rather take advantage of the connection between the observability inequality for each problem and the spectral properties of structured matrices. Alternative proofs are hence exposed owing to algebraic estimates developed in the previous section. As a result, we bring about easier proofs than those using Carleman estimates (see $[11,17]$ ) and provide a way to evaluate the ill-conditioning of the observability problem to approximate. A the end, Section ?? is dedicated to the cost of short observation for two of the three problems we are interested in.

Notation - Let $\ell^{2}(\mathbb{R})$ be the Hilbert space of countably infinite real sequence $\boldsymbol{\psi}=\left(\psi_{k}\right)_{k \geq 1}$ that are square summable and denote its norm by $\|\cdot\|_{\ell^{2}}$. For a given integer $N>1$, we will use the symbol $\ell_{N}^{2}(\mathbb{R})$ for the sub-space in $\ell^{2}(\mathbb{R})$ involving the sequence $\boldsymbol{\psi}$ whose entries $\left(\psi_{k}\right)_{k \geq N+1}$ vanish. It is isomorphic to the standard space $\mathbb{R}^{N}$ endowed with the Euclidean norm. The closure of the union of the subspaces $\ell_{N}^{2}(\mathbb{R})$ is dense in the whole space $\ell^{2}(\mathbb{R})$. Next, we consider a 
countably infinite matrix $\mathcal{C}$, defined by the real entries $\left(c_{k, m}\right)_{k, m \geq 1}$. Throughout, the notation $\mathcal{C}_{N}^{(T)}$ stands for the principle sub-matrix with order $N$ of $\mathcal{C}$. It may be identified to the infinite matrix

$$
\mathcal{C}_{N}^{(T)}=\left(\begin{array}{ccc}
\left(c_{k, m}\right)_{1 \leq k, m \leq N} & 0 & \cdots \\
0 & 0 & \cdots \\
\vdots & \vdots & \ddots
\end{array}\right)
$$

If $\mathcal{P}_{N}$ stands for the canonical projection operator from $\ell^{2}(\mathbb{R})$ into $\ell_{N}^{2}(\mathbb{R})$ (equivalently on $\mathbb{R}^{N}$ ), we may write that $\mathcal{C}_{N}^{(T)}=\mathcal{P}_{N} \mathcal{C} \mathcal{P}_{N}$. Subsequently, we shall accept, in some places, a notation abuse concerning the principal sub-matrices of $\mathcal{C}_{N}^{(T)}$ with dimension $N$. It has either the form of (1), an infinite matrix representing an operator defined on $\ell_{N}^{2}(\mathbb{R})$, or simply the square matrix $\left(c_{k, m}\right)_{1 \leq k, m \leq N}$ with dimension $N$ related to a linear application in $\mathbb{R}^{N}$. We refer to [9] for the fundamental properties of these spaces. Now, for any separable Banach space $X$ provided with the norm $\|\cdot\|_{X}$, we denote by $L^{2}(0, T ; X)$ the space of measurable functions $v$ from $(0, t)$ in $X$ such that

$$
\|v\|_{L^{2}(0, T ; X)}=\left(\int_{0}^{T}\|v(\cdot, s)\|_{X}^{2} d s\right)^{1 / 2}<+\infty .
$$

We also use the space $\mathscr{C}(0, T ; X)$ of continuous functions $v$ from $[0, T]$ in $X$.

\section{Infinite Cauchy matrices}

We study some countably infinite matrices related to some observability problems for the heat equation. The aim is to derive useful results on their spectra. Infinite matrices cannot be treated crudely and their handling requires a minimum of care (see [2]). We are here concerned with some of these matrices that are representation of some linear operators defined on $\ell^{2}(\mathbb{R})$.

Let $\left(\lambda_{k}\right)_{k \geq 1}$ be a positive increasing sequence of real numbers $\left(0<\lambda_{1}<\lambda_{2}<\right.$ $\left.\lambda_{3}<\cdots\right)$ and $T>0$ be a given positive real number. We define the symmetric Pick matrix $\mathcal{C}^{(T)}$ by the entries

$$
\left(c^{(T)}\right)_{k, m}=\frac{1-e^{-\lambda_{k} T} e^{-\lambda_{m} T}}{\lambda_{k}+\lambda_{m}}, \quad \forall k, m \geq 1 .
$$

The particular matrix $\mathcal{C}(=\mathcal{C})$ whose coefficients are reduced to

$$
c_{k, m}=\frac{1}{\lambda_{k}+\lambda_{m}}, \quad \forall k, m \geq 1
$$


is a Cauchy matrix and will play a central role here. Introducing the diagonal matrix $\mathcal{Q}^{(T)}=\operatorname{diag}\left(e^{-\lambda_{k} T}\right)_{k \geq 1}$, we split $\mathcal{C}^{(T)}$ into two Cauchy matrices

$$
\mathcal{C}^{(T)}=\mathcal{C}-\mathcal{Q}^{(T)} \mathcal{C} \mathcal{Q}^{(T)}
$$

Throughout, we focus on the class of matrices $\mathcal{C}^{(T)}$ constructed through a sequence $\left(\lambda_{k}\right)_{k \geq 1}$ satisfying

$$
\sum_{k \geq 1} \frac{1}{\left(\lambda_{k}\right)^{\varrho}}<\infty
$$

for some real number $\varrho \in] 0,1\left[\right.$. The summability (2) tells in particular that $\left(\lambda_{k}\right)_{k \geq 1}$ grows to infinity.

Remark 2.1 The real number $\varrho_{*}$ defined as the infimum of all @ for which assumption (2) is satisfied is called the summation exponent of the sequence $\left(\lambda_{k}\right)_{k \geq 1}$. The sum in (2) may be finite or not for $\varrho=\varrho_{*}$.

The spectrum of the Pick matrix $\mathcal{C}^{(T)}$ is the cornerstone of Section 3 treating of the observability of diffusion equations. We are hence primarily interested in studying $\mathcal{C}^{(T)}$. The way to proceed consists in checking out, by means of Remez' theorem, the equivalence between $\mathcal{C}^{(T)}$ and the Cauchy matrix $\mathcal{C}$. Then, we prove the desired results on $\mathcal{C}$ which extend to $\mathcal{C}^{(T)}$ as well. The notations $\mathcal{C}$ and $\mathcal{C}^{(T)}$ will be used to point out either the matrices themselves or their counterpart operators in $\ell^{2}(\mathbb{R})$.

Lemma 2.2 Assume that (2) holds. Then $\mathcal{C}$ is compact, self-adjoint and nonnegative with $\mathcal{N}(\mathcal{C})=\{0\}$. The range $\mathcal{R}(\mathcal{C})$ is not closed and $\overline{\mathcal{R}(\mathcal{C})}=\ell^{2}(\mathbb{R})$.

Proof. Using (2), we obtain a bound on the Frobenius norm of $\mathcal{C}$

$$
\|\mathcal{C}\|_{F}^{2}=\sum_{k \geq 1} \sum_{m \geq 1} \frac{1}{\left(\lambda_{k}+\lambda_{m}\right)^{2}} \leq \frac{1}{4}\left[\sum_{k \geq 1} \frac{1}{\lambda_{k}}\right]\left[\sum_{m \geq 1} \frac{1}{\lambda_{m}}\right]=\frac{1}{4}\left[\sum_{k \geq 1} \frac{1}{\lambda_{k}}\right]^{2}<\infty .
$$

Consequently $\mathcal{C}$ is a Hilbert-Schmidt operator and is by then compact (see [5]). Now, let $\varphi=\left(\varphi_{k}\right)_{k \geq 1}$ be in $\ell^{2}(\mathbb{R})$ and define the function

$$
\varphi(t)=\sum_{k \geq 1} \varphi_{k} e^{-\lambda_{k} t}, \quad \forall t \geq 0
$$


Easy computations yield

$$
\|\varphi\|_{L^{2}(0, \infty)}^{2}=(\mathcal{C} \boldsymbol{\varphi}, \boldsymbol{\varphi})_{\ell^{2}(\mathbb{R})} \geq 0 .
$$

Hence, $\varphi \in \mathcal{N}(\mathcal{C})$ implies $\varphi=0$. Since the sequence $\left(1 / \lambda_{k}\right)_{k \geq 1}$ is summable, by Müntz-Szász theorem (see [32]), the family $\left(e^{-\lambda_{k} t}\right)_{m \geq 1}$ is linearly independent in $L^{2}(0, \infty)$. Consequently, $\varphi_{k}=0, \forall k \geq 1$, and the kernel of $\mathcal{C}$ is reduced to the trivial subspace; i.e. $\mathcal{N}(\mathcal{C})=\{0\}$. According to the symmetry of $\mathcal{C}$, we derive that $\overline{\mathcal{R}(\mathcal{C})}=\mathcal{N}(\mathcal{C})^{\perp}=\ell^{2}(\mathbb{R})$. The range cannot be closed otherwise by the open mapping theorem $\mathcal{C}$ would be isomorphic. This contradicts the compactness of $\mathcal{C}$.

Lemma 2.3 Under assumption (2), $\mathcal{C}^{(T)}$ is compact, self-adjoint and non-negative with $\mathcal{N}\left(\mathcal{C}^{(T)}\right)=\{0\}$ and $\overline{\mathcal{R}\left(\mathcal{C}^{(T)}\right)}=\ell^{2}(\mathbb{R})$.

Proof. The proof for Pick's matrix $\mathcal{C}^{(T)}$ follows the same arguments as the one for Cauchy's matrix $\mathcal{C}$ and is hence skipped over.

The spectral equivalence between operators $\mathcal{C}^{(T)}$ and $\mathcal{C}$ is at the basis of the forthcoming developments. From now on we write $\mathcal{C}^{(T)} \leq \mathcal{C}$ to express Lowner partial ordering:

$$
\left(\mathcal{C}^{(T)} \boldsymbol{\varphi}, \boldsymbol{\varphi}\right)_{\ell^{2}} \leq(\mathcal{C} \boldsymbol{\varphi}, \boldsymbol{\varphi})_{\ell^{2}}, \quad \forall \boldsymbol{\varphi} \in \ell^{2}(\mathbb{R})
$$

Lemma 2.4 Under assumption (2), there exists $\left.\nu_{T} \in\right] 0,1[$ such that

$$
\nu_{T} \mathcal{C} \leq \mathcal{C}^{(T)} \leq \mathcal{C}
$$

Moreover, we have

$$
\lim _{T \rightarrow 0} \frac{\nu_{T}}{T}=0
$$

Proof. Note that a less sharp bound on $\nu_{T}$ can be can easily obtained

$$
\nu_{T} \leq 1-e^{-2 \lambda_{1} T} \leq 2 \lambda_{1} T, \quad \forall T>0 .
$$

The non-negative definiteness of matrix $\mathcal{Q}^{(T)} \mathcal{C} \mathcal{Q}^{(T)}$, directly ensued from the definiteness of $\mathcal{C}$, gives the right hand side of the inequality. The other inequality is a consequence of a particular variant of the Remez type inequality in a Müntz subspace of $L^{2}(0, \infty)$, namely the one spanned by the non-complete exponential 
system $\left(e^{-\lambda_{k} t}\right)_{k \geq 1}$. On account of assumption (2), Theorem 5.6 of [4] applies. Hence, there exists a constant $\gamma_{T}$ that depends only on $T$ such that

$$
\|\varphi\|_{L^{2}(0, \infty)} \leq \gamma_{T}\|\varphi\|_{L^{2}(0, T)}
$$

for all $\varphi$ defined by (4). We have necessarily that $\gamma_{T}>1$. This inequality is equivalent to the left hand side of the inequality in the Lemma with $\nu_{T}=\left(\gamma_{T}\right)^{-2}$. For $\varphi \in \ell_{N}^{2}(\mathbb{R}), \varphi \neq 0$, we deduce from

$$
\nu_{T}(\mathcal{C} \varphi, \varphi) \leq\left(\mathcal{C}^{(T)} \boldsymbol{\varphi}, \boldsymbol{\varphi}\right)
$$

that

$$
\frac{\nu_{T}}{T} \leq \frac{\left(\mathcal{J}_{N} \varphi, \varphi\right)}{(\mathcal{C} \varphi, \varphi)}
$$

where $\mathcal{J}_{N}$ is the square matrix whose entries are all equal to one. It is a rank-one matrix, the dimension of its kernel is $(N-1)$. Choosing $\varphi$ in $\mathcal{N}\left(\mathcal{J}_{N}\right)$ shows that the limit of $\nu_{T} / T$ is necessarily zero. The proof is complete.

We now derive a spectral comparison between the operators $\mathcal{C}^{(T)}$ and $\left(\mathcal{Q}^{(T)}\right)^{2}(=$ $\left.\mathcal{Q}^{(2 T)}\right)$. Another sufficient assumption is now required on the sequence $\left(\lambda_{k}\right)_{k \geq 1}$ (regularly increasing sequence):

$$
\inf _{k \geq 1}\left(\lambda_{k+1}-\lambda_{k}\right)>0 .
$$

The proof of the following is the key milestone of Section 3.

Proposition 2.5 Under assumptions (2) and (6), there exists a constant $\eta_{T}>0$ such that

$$
\mathcal{C}^{(T)} \geq \eta_{T}\left(\mathcal{Q}^{(T)}\right)^{2}
$$

Proof. On account of the spectral equivalence in Lemma 2.4, it is sufficient to show the estimate for the Cauchy matrix $\mathcal{C}$. That result will be established if it is proved for the truncated operators $\mathcal{C}_{N}$ and $\mathcal{Q}_{N}^{(T)}$ with a constant $\eta_{T}$ that is independent of $N$. Indeed, it is clear that the Frobenius norms of $\left(\mathcal{C}-\mathcal{C}_{N}\right)$ and $\left(\mathcal{Q}^{(T)}-\mathcal{Q}_{N}^{(T)}\right)$ decay to zero as $N$ grows. We deduce that $\mathcal{C}_{N}$ and $\mathcal{Q}_{N}^{(T)}$ converge respectively toward $\mathcal{C}$ and $\mathcal{Q}^{(T)}$ with respect to the norm of operators in $\ell^{2}(\mathbb{R})$. After these preliminaries and with the notation abuse mentioned in the introduction, we are going to show that

$$
\left(\mathcal{C}_{N} \varphi, \varphi\right)_{\mathbb{R}^{N}} \geq \eta_{T}\left\|\mathcal{Q}_{N}^{(T)} \varphi\right\|_{\mathbb{R}^{N}}^{2}, \quad \forall \varphi \in \mathbb{R}^{N}
$$


Recall that $\mathcal{C}_{N}$ and $\mathcal{Q}_{N}^{(T)}$ may be viewed as square matrices of dimension $N$. The desired estimate is achieved if we prove that $\mathcal{Q}_{N}^{(T)}\left(\mathcal{C}_{N}\right)^{-1} \mathcal{Q}_{N}^{(T)}$ is spectrally bounded uniformly in $N$. The clue is the existence of a closed form of the inverse of the Cauchy matrix

$$
\left(\mathcal{C}_{N}\right)^{-1}=\mathcal{D}_{N} \mathcal{C}_{N} \mathcal{D}_{N}
$$

with $\mathcal{D}_{N}$ the diagonal matrix whose (diagonal) entries are (see [31, 23])

$$
d_{N, k}=2 \lambda_{k} \prod_{\substack{1 \leq m \leq N \\ m \neq k}} \frac{\lambda_{m}+\lambda_{k}}{\lambda_{m}-\lambda_{k}}=2 \lambda_{k} \prod_{\substack{1 \leq m \leq N \\ m \neq k}} \frac{1+\frac{\lambda_{k}}{\lambda_{m}}}{1-\frac{\lambda_{k}}{\lambda_{m}}} .
$$

Consequently

$$
\mathcal{Q}_{N}^{(T)}\left(\mathcal{C}_{N}\right)^{-1} \mathcal{Q}_{N}^{(T)}=\left(\mathcal{Q}_{N}^{(T)} \mathcal{D}_{N}\right) \mathcal{C}_{N}\left(\mathcal{D}_{N} \mathcal{Q}_{N}^{(T)}\right)
$$

Since matrix $\mathcal{C}_{N}$ is uniformly bounded in $N$ (see (3)), it remains to prove that $\mathcal{Q}_{N}^{(T)} \mathcal{D}_{N}$ is also uniformly bounded. Due to assumptions (2) and (6), a bound on $\mathcal{D}_{N}$ is given by

$$
\left|d_{N, k}\right| \leq\left|d_{\infty, k}\right| \leq K e^{\zeta\left(\lambda_{k}\right)^{\varrho}}, \quad(\forall k \geq 1)
$$

where $K$ is a positive real number depending on $\zeta>0$ and blowing up as $\zeta$ tends toward zero. The exponent $\varrho>\varrho_{*}$ is the one appearing in (2). This result can be obtained from [3, Chapitre IV]. It comes from the second minimization theorem on entire functions stated by J. Hadamard in [13], re-investigated and improved by different authors. L. Schwarz used it repeatedly in the form given here when $\varrho=1$ (see [32, page 32, formula $\left.\left(9 \mathrm{~h}^{\prime}\right)\right]$ ). The uniform boundedness of the operator $\left(\mathcal{Q}_{N}^{(T)} \mathcal{D}_{N}\right)=\operatorname{diag}\left(e^{-\lambda_{k} T} d_{N, k}\right)_{1 \leq k \leq N}$ results from

$$
e^{-\lambda_{k} T}\left|d_{N, k}\right| \leq K e^{-\lambda_{k} T+\zeta\left(\lambda_{k}\right)^{\varrho}}, \quad \forall k \geq 1 .
$$

Given that $\varrho<1$, the sequence in the right side is uniformly bounded and so is $\left(e^{-\lambda_{k} T} d_{N, k}\right)_{k}$. We obtain finally that

$$
\left(\mathcal{Q}_{N}^{(T)}\left(\mathcal{C}_{N}\right)^{-1} \mathcal{Q}_{N}^{(T)} \boldsymbol{\varphi}, \boldsymbol{\varphi}\right)_{\mathbb{R}^{N}} \leq \mu_{T}\|\varphi\|_{\mathbb{R}^{N}}^{2}, \quad \forall \boldsymbol{\varphi} \in \mathbb{R}^{N}
$$

The constant $\mu_{T}$ does not depend on $N$. The proof of (7) is therefore complete with $\eta_{T}=\nu_{T} / \mu_{T}$. 


\section{Observability of diffusion equations}

We revisit different observability results for the diffusion equation. These observability results are important for null-controllability problems (see [30, 20, 17, 11, 27, ?, 36, 34, 1] and references therein). The methodology is based on Fourier series already used in many works. We provide alternative proofs to those developed for instance in $[10,8,24,12]$ replacing the study of bi-orthogonal sequences by algebraic arguments.

Let $I$ be the segment $(0, \pi)$ of the real axis and $T>0$ be a fixed real number. We set $Q=I \times] 0, T[$. The generic point in $I$ is denoted by $x$ and the generic time is $t$. For a given initial state $\psi \in L^{2}(I)$, we denote by $q_{\psi}$ the unique solution of the diffusion problem

$$
\begin{aligned}
\partial_{t} q_{\psi}-\left(a(x) q_{\psi}^{\prime}\right)^{\prime}+b(x) q_{\psi} & =0 & & \text { in } Q, \\
q_{\psi}(0, t)=0, & q_{\psi}(\pi, t)=0 & & \forall t \in(0, T), \\
q_{\psi}(x, 0) & =\psi & & \forall x \in I .
\end{aligned}
$$

The symbol' is used for the space derivative $\partial_{x}$. The reaction coefficient $b(\cdot) \in$ $L^{\infty}(I)$ is assumed non-negative for simplicity, i.e., $b(x) \geq 0, \forall x \in I$ and the conductivity parameter $a(\cdot) \in L^{\infty}(I)$ is supposed to be positive and bounded away from zero. This means that $a(x) \geq a_{*}, \forall x \in I$, for some real constant $a_{*}>0$. This diffusion problem has a unique solution $q_{\psi}$ in $L^{2}\left(0, T ; H_{0}^{1}(I)\right) \cap$ $\mathscr{C}\left([0, T] ; L^{2}(I)\right)$ (see [22, Chap. 4]).

\subsection{First observability problem}

The first problem is picked-up from [8]. Let $v$ be a given function in $L^{2}(I)$. For any $\psi=\psi(x)$ in $L^{2}(I)$, we set

$$
(B \psi)(t)=-\left(q_{\psi}(t), v\right)_{L^{2}(I)}, \quad \forall t \in(0, T) .
$$

The observability inequality we are concerned with can be formulated as follows

$$
\left\|q_{\psi}(T)\right\|_{L^{2}(I)} \leq \alpha_{T}\|B \psi\|_{L^{2}(0, T)}, \quad \forall \psi \in L^{2}(I),
$$

with a constant $\alpha_{T}$ expected to depend on $T$.

Analysis of the observability operator is necessary for null-controllability of the diffusion problem with a source control expressed by $c(t, x)=f(t) v(x)$ in 
$Q$ where $v \in L^{2}(I)$ is a given function and $f(t) \in L^{2}(0, T)$ is the control (see [8]). Success or failure for HUM method in proving existence of a control $c$ is subordinated to the validity or not of estimate (12).

In [8], S. Dolecki proved the existence of an optimal time $T_{*}$ such that if $T>T_{*}$, the observability holds at this time $T$ and, if $T<T_{*}$, the observability fails at time $T$. We aim at reaching the same conclusion following a different strategy.

To step forth, we need to put (12) under a (infinite) matrix form. We consider therefore an orthonormal basis $\left(e_{k}(\cdot)\right)_{k \geq 1}$ in $L^{2}(I)$ of eigenfunctions of the autoadjoint operator

$$
\psi \mapsto-\left(a(x) \psi^{\prime}\right)^{\prime}+b(x) \psi
$$

defined on $H^{2} \cap H_{0}^{1}(I)$. The eigenvalues $\left(\lambda_{k}\right)_{k \geq 1}$ are all simple and positive. They are ordered increasingly. Actually, following [7, Chapter VI], we have the following asymptotics : there exist real numbers $\beta$ and $\tau>0$ depending on $a(\cdot)$ and a bounded sequence $\left(\varepsilon_{k}\right)_{k \geq 1}$ such that

$$
\frac{\lambda_{k}}{(k+\beta)^{2}}=\tau+\frac{\varepsilon_{k}}{k^{2}} \quad \forall k \geq 1 .
$$

This indicates in particular that assumption (2) is fulfilled for any $\varrho>\varrho_{*}=1 / 2$ and so is hypothesis (6).

Remark 3.1 A sharp bound of the sequences $\left(d_{N, k}\right)_{k}$ and $\left(d_{\infty, k}\right)_{k}$ defined in (9) is provided in [10], that is

$$
\left|d_{N, k}\right| \leq\left|d_{\infty, k}\right| \leq K \lambda_{k} e^{\zeta\left(\lambda_{k}\right)^{1 / 2}}, \quad \forall k \geq 1 .
$$

To derive a useful expression of $B$, we follow [10, 27]. Let $\psi$ be given in $L^{2}(I)$ represented by its Fourier expansion,

$$
\psi(x)=\sum_{k \geq 1} \psi_{k} e_{k}(x) \quad \text { in } I .
$$

Then, $B$ can be expressed as follows

$$
(B \psi)(t)=-\sum_{k \geq 1}\left(v_{k} e^{-\lambda_{k} t}\right) \psi_{k},
$$

where $v_{k}=\left(v, e_{k}\right)_{L^{2}(I)}$ are the Fourier coefficients of $v$. Next, straightforward calculations produce a new form of the observability estimate (12), using infinite matrices

$$
\eta_{T}\left\|\mathcal{Q}^{(T)} \boldsymbol{\psi}\right\|_{\ell^{2}}^{2} \leq\left(\mathcal{C}^{(T)} \mathcal{V} \boldsymbol{\psi}, \mathcal{V} \boldsymbol{\psi}\right)_{\ell^{2}}, \quad \forall \boldsymbol{\psi} \in \ell^{2}(\mathbb{R})
$$


with $\mathcal{V}$ the diagonal operator $\mathcal{V}=\operatorname{diag}\left(v_{k}\right)_{k \geq 1}$ and $\eta_{T}=\left(\alpha_{T}\right)^{-2}$. The following result holds.

Theorem 3.2 For a given $v \in L^{2}(I)$, assume that

$$
\sup _{k \geq 1} \frac{e^{-\lambda_{k} T_{*}}}{\left|v_{k}\right|}<\infty
$$

Then, for any $T>T_{*}$, there exists a constant $\eta_{T}>0$ such that

$$
\mathcal{V} \mathcal{C}^{(T)} \mathcal{V} \geq \eta_{T}\left(\mathcal{Q}^{(T)}\right)^{2}
$$

As a consequence the observability estimate (12) holds true.

Proof. According to Lemma 2.4, we have

$$
\mathcal{V} \mathcal{C}^{(T)} \mathcal{V} \geq \nu_{T} \mathcal{V C V}
$$

If we establish the observation (16) for $\mathcal{C}$ we can afterwards derive it for $\mathcal{C}^{(T)}$, since the only new effect is to multiply $\eta_{T}$ by $\nu_{T}$. Observe now that the Frobenius norm of (infinite) matrix $\mathcal{V}$ coincides with $\|v\|_{L^{2}(I)}$. It is therefore finite. The truncated operator $\mathcal{V}_{N}=\operatorname{diag}\left(v_{k}\right)_{1 \leq k \leq N}$ converges toward $\mathcal{V}$, with respect to the norm of operators in $\ell^{2}(\mathbb{R})$. Consequently, to derive the observability estimate (16) we can replace the operators $\mathcal{V}, \mathcal{C}$ and $\mathcal{Q}^{(T)}$ by their truncated counterparts $\mathcal{V}_{N}, \mathcal{C}_{N}$ and $\mathcal{Q}_{N}^{(T)}$ provided that $\eta_{T}$ is independent of $N$. We follow the same arguments as those that yielded inequality (11). Thus we are expected to show that the finite order matrix

$$
\mathcal{Q}_{N}^{(T)}\left(\mathcal{V}_{N} \mathcal{C}_{N} \mathcal{V}_{N}\right)^{-1} \mathcal{Q}_{N}^{(T)}
$$

is uniformly bounded with respect to $N$. Using (8) we rewrite the matrix as

$$
\mathcal{Q}_{N}^{(T)}\left(\mathcal{V}_{N} \mathcal{C}_{N} \mathcal{V}_{N}\right)^{-1} \mathcal{Q}_{N}^{(T)}=\left[\mathcal{Q}_{N}^{(T)}\left(\mathcal{V}_{N}\right)^{-1} \mathcal{D}_{N}\right] \mathcal{C}_{N}\left[\mathcal{D}_{N}\left(\mathcal{V}_{N}\right)^{-1} \mathcal{Q}_{N}^{(T)}\right]
$$

Let us derive a uniform bound for $\left(\mathcal{Q}_{N}^{(T)}\left(\mathcal{V}_{N}\right)^{-1} \mathcal{D}_{N}\right)=\operatorname{diag}\left(e^{-\lambda_{k} T} \frac{d_{N, k}}{v_{k}}\right)_{1 \leq k \leq N}$. Owing to (13) we have for all $k \geq 1$,

$$
e^{-\lambda_{k} T} \frac{\left|d_{N, k}\right|}{\left|v_{k}\right|} \leq K \frac{\lambda_{k} e^{-\lambda_{k} T+\zeta\left(\lambda_{k}\right)^{1 / 2}}}{\left|v_{k}\right|} \leq K\left(\lambda_{k} e^{-\lambda_{k}\left(T-T_{*}\right)+\zeta\left(\lambda_{k}\right)^{1 / 2}}\right) \frac{e^{-\lambda_{k} T_{*}}}{\left|v_{k}\right|} .
$$

Under assumption (15), the right sequence can be bounded by a constant that does not depend on $N$. The proof is complete. 
We can also establish the non-observability result given in [8, Theorem 1].

Lemma 3.3 Assume that, $v_{k} \neq 0, \forall k \geq 1$ and that, for some $T_{*}$,

$$
\sum_{k \geq 1} \frac{e^{-\lambda_{k} T_{*}}}{\left|v_{k}\right|}=\infty
$$

Then, the observability (12) fails for all $T<T_{*}$.

Proof. Cauchy-Schwarz inequality and hypothesis (17) provide straightforwardly that

$$
\sum_{k \geq 1} \frac{e^{-2 \lambda_{k} T}}{\left(v_{k}\right)^{2}}=\infty
$$

for all $T<T_{*}$. Next, we consider $\boldsymbol{\psi}=\left(\frac{e^{-\lambda_{k} \delta}}{v_{k}}\right)_{1 \leq k \leq N}$, for some $\delta>0$ to be chosen later on. Direct computations yield in one hand

$$
\left\|\mathcal{Q}^{(T)} \boldsymbol{\psi}\right\|_{\ell^{2}}^{2}=\sum_{1 \leq k \leq N} \frac{e^{-2 \lambda_{k}(T+\delta)}}{\left(v_{k}\right)^{2}}
$$

and this sum blows up for large $N$, as soon as $T+\delta<T_{*}$. In the other hand, boundedness (by unity) of $\mathcal{C}^{(T)}$ leads to

$$
\left(\mathcal{C}^{(T)} \mathcal{V} \boldsymbol{\psi}, \mathcal{V} \boldsymbol{\psi}\right)_{\ell^{2}} \leq \sum_{1 \leq k \leq N} e^{-2 \lambda_{k} \delta}
$$

and this is bounded uniformly in $N$. In conclusion, the failure of (12) is illustrated via (14).

Remark 3.4 As noticed in [8, Corollary 1], the time of observation is tightly connected to $v$; it is given by the formula

$$
T_{*}=\limsup _{k \rightarrow \infty}\left(-\frac{\ln \left|v_{k}\right|}{\lambda_{k}}\right) .
$$

\subsection{Boundary observability}

This boundary observability problem is connected to the null-controllability of the diffusion equation by a Dirichlet control which is activated, let us say at $x=\pi$. The corresponding observability operator is then defined by

$$
(D \psi)(t)=a(\pi) q_{\psi}^{\prime}(t, \pi), \quad \forall t \in(0, T) .
$$


Viewed as mapping $L^{2}(I)$ into $L^{2}(0, T)$, the operator $D$ turns out to be unbounded with a dense domain and it is closed. This brings a little trouble and some care is to be paid in the analysis. The observability estimate may be written as (see $[17,24])$

$$
\left\|q_{\psi}(T)\right\|_{L^{2}(I)} \leq \alpha_{T}\|D \psi\|_{L^{2}(0, T)}, \quad \forall \psi \in \mathbb{D}(D) .
$$

Put under a matrix form, it is transformed into

$$
\left(\mathcal{C}^{(T)} \mathcal{W} \boldsymbol{\psi}, \mathcal{W} \boldsymbol{\psi}\right)_{\ell^{2}} \geq \eta_{T}\left\|\mathcal{Q}^{(T)} \boldsymbol{\psi}\right\|_{\ell^{2}}^{2}, \quad \forall \boldsymbol{\psi} \in \mathbb{D}(D) .
$$

The diagonal operator $\mathcal{W}=\operatorname{diag}\left(w_{k}\right)_{k \geq 1}$ with entries $w_{k}=a(\pi) e_{k}^{\prime}(\pi)$ is unbounded. Indeed, according to the estimate on the eigenfunctions $\left(e_{k}\right)_{k \geq 1}$ established by T. I. Seidman (see [33, Theorem 2.4]), we have

$$
\left|\left(a e_{k}^{\prime}\right)(\pi)\right| \geq \gamma\left(\lambda_{k}\right)^{1 / 2}, \quad \forall k \geq 1,
$$

for a constant $\gamma>0$. This operator has nevertheless some advantageous features. Its domain is dense with the inclusion $\mathbb{D}(\mathcal{W}) \subset \mathbb{D}(D)$. Besides, $\mathcal{W}^{-1}$ is a HilbertSchmidt operator since its Frobenius norm is finite:

$$
\left\|\mathcal{W}^{-1}\right\|_{F} \leq \frac{1}{\gamma}\left[\sum_{k \geq 1} \frac{1}{\lambda_{k}}\right]^{1 / 2}<\infty .
$$

Hence $\mathcal{W}^{-1}$ is a compact operator. Let us now write the observability inequality (18) as

$$
\left(\mathcal{C}^{(T)} \boldsymbol{\psi}, \boldsymbol{\psi}\right)_{\ell^{2}} \geq \eta_{T}\left\|\mathcal{Q}^{(T)} \mathcal{W}^{-1} \boldsymbol{\psi}\right\|_{\ell^{2}}^{2}, \quad \forall \boldsymbol{\psi} \in \ell^{2}(\mathbb{R}) .
$$

The observability estimate (18) is true since we have the following.

Theorem 3.5 There exists a constant $\eta_{T}>0$ such that

$$
\mathcal{C}^{(T)} \geq \eta_{T}\left[\mathcal{Q}^{(T)} \mathcal{W}^{-1}\right]^{2}
$$

Proof. The proof is but a duplication of the previous one. The important issue is to check out whether the truncation of the operators $\mathcal{C}^{(T)}($ or $\mathcal{C}), \mathcal{Q}^{(T)}$ and $\mathcal{W}^{-1}$ converge toward their corresponding full operators. This is guaranteed by the finiteness of their Frobenius norms. Analogous algebraic manipulations, in particular the explicit inverse of $\mathcal{C}_{N}^{(T)}$, transform the issue into showing the following bound

$$
\left(\mathcal{C}_{N}^{(T)}\left[\mathcal{Q}_{N}^{(T)}\left(\mathcal{W}_{N}\right)^{-1} \mathcal{D}_{N}\right] \boldsymbol{\varphi},\left[\mathcal{Q}_{N}^{(T)}\left(\mathcal{W}_{N}\right)^{-1} \mathcal{D}_{N}\right] \varphi\right)_{\mathbb{R}^{N}} \leq \mu_{T}\|\boldsymbol{\varphi}\|_{\mathbb{R}^{N}}^{2}, \quad \forall \boldsymbol{\varphi} \in \mathbb{R}^{N}
$$


After using (13), the only point that remains to inspect is the uniform boundedness of

$$
e^{-\lambda_{k} T} \frac{\left|d_{N, k}\right|}{\left|w_{k}\right|} \leq K\left(\lambda_{k}\right)^{1 / 2} e^{-\lambda_{k} T+\zeta\left(\lambda_{k}\right)^{1 / 2}}, \quad \forall k \geq 1 .
$$

This result holds true. The proof is complete with $\eta_{T}=\frac{1}{\mu_{T}}$.

Remark 3.6 It is readily checked out that $\left(\mathcal{Q}_{N}^{(T)} \mathcal{D}_{N}\right)$ strongly converges towards $\left(\mathcal{Q}^{(T)} \mathcal{D}\right)$. As a result, it is possible to pass to the limit in estimate (19). We have therefore

$$
\left(\mathcal{C}\left[\left(\mathcal{Q}^{(T)} \mathcal{D}\right) \mathcal{W}^{-1}\right] \boldsymbol{\varphi},\left[\left(\mathcal{Q}^{(T)} \mathcal{D}\right) \mathcal{W}^{-1}\right] \boldsymbol{\varphi}\right)_{\ell^{2}(\mathbb{R})} \leq \mu_{T}\|\boldsymbol{\varphi}\|_{\ell^{2}(R)}^{2}, \quad \forall \boldsymbol{\varphi} \in \ell^{2}(\mathbb{R})
$$

This inequality makes sense mathematically. In fact, the composition of the bounded operator $\mathcal{Q}^{(T)}$ and the unbounded one $\mathcal{D}$ results in a bounded operator denoted here by $\left(\mathcal{Q}^{(T)} \mathcal{D}\right)$. Estimate (20) is also equivalent to the observation estimate (18).

\subsection{Distributed observability}

Let us denote an arbitrary sub-segment $\omega$ of $I$, with $\omega \neq I$. The observability operator required for the distributed null-controllability of the diffusion equation with a control supported in $\omega$ is defined as follows

$$
(G \psi)(t, x)=q_{\psi}(t, x) \chi_{\omega}(x) \quad \forall(t, x) \in Q,
$$

with $\chi_{\omega}$ the characteristic function of $\omega$. Once again the observability estimate can be expressed as

$$
\left\|q_{\psi}(T)\right\|_{L^{2}(I)} \leq \alpha_{T}\|G \psi\|_{L^{2}(Q)}, \quad \forall \psi \in L^{2}(I) .
$$

To transform this inequality into an algebraic formula, we need some further definitions and notations. We introduce the (infinite) Gramian matrix

$$
\left.\mathcal{U}=\left(u_{k, m}\right)_{k, m \geq 1}=\left(\left(e_{k}, e_{m}\right)_{L^{2}(\omega)}\right)\right)_{k, m \geq 1} .
$$

It is the matrix representation, on the Hilbert basis $\left(e_{k}\right)_{k \geq 1}$, of the multiplication operator in $L^{2}(I)$ by the characteristic function $\chi_{\omega} . \mathcal{U}$ is symmetric and nonnegative definite; it is a bounded operator as it determines a contraction in $\ell^{2}(\mathbb{R})$.

$$
(\mathcal{U} \boldsymbol{\psi}, \boldsymbol{\psi})_{\ell^{2}} \leq\|\boldsymbol{\psi}\|_{\ell^{2}}^{2}, \quad \forall \boldsymbol{\psi} \in \ell^{2}(\mathbb{R})
$$


Owing to Cauchy-Schwarz inequality one easily sees that

$$
\left|u_{k, m}\right|=\left|\left(e_{k}, e_{m}\right)_{L^{2}(\omega)}\right| \leq 1, \quad \forall k, m \geq 1 .
$$

Writing the observability inequality (21) in algebraic terms results in

$$
\eta_{T}\left\|\mathcal{Q}^{(T)} \boldsymbol{\psi}\right\|_{\ell^{2}}^{2} \leq\left(\mathcal{C}^{(T)} \circ \mathcal{U} \boldsymbol{\psi}, \boldsymbol{\psi}\right)_{\ell^{2}}, \quad \forall \boldsymbol{\psi} \in \ell^{2}(\mathbb{R})
$$

The operation $\circ$ stands for the Hadamard (or Schur) entrywise product of matrices (see $[14,35])$. The entries of this product are hence determined as

$$
\mathcal{C}^{(T)} \circ \mathcal{U}=\left(\left(c^{(T)}\right)_{k, m} u_{k, m}\right)_{k, m \geq 1}
$$

This fixed, the following theorem holds.

Theorem 3.7 There exists a constant $\eta_{T}>0$ such that

$$
\mathcal{C}^{(T)} \circ \mathcal{U} \geq \eta_{T}\left(\mathcal{Q}^{(T)}\right)^{2}
$$

Hence, the observability estimate (21) holds true.

Proof. It follows the same conducting thread as the previous theorems. First and foremost we explain why we may work with the truncated matrices. We start by noticing that, due to the non-negative definiteness of $\mathcal{U}$, the inequality $\nu_{T} \mathcal{C} \leq \mathcal{C}^{(T)}$ implies that (see [35, Theorem 6.1])

$$
\nu_{T}(\mathcal{C} \circ \mathcal{U}) \leq \mathcal{C}^{(T)} \circ \mathcal{U}
$$

We will thus replace $\mathcal{C}^{(T)}$ by $\mathcal{C}$. Then, it is obvious that $\mathcal{C}_{N} \circ \mathcal{U}_{N}=\mathcal{C}_{N} \circ \mathcal{U}$. Accounting for the bound (22), we directly issue that

$$
\left\|\mathcal{C} \circ \mathcal{U}-\mathcal{C}_{N} \circ \mathcal{U}_{N}\right\|_{F}=\left\|\left(\mathcal{C}-\mathcal{C}_{N}\right) \circ \mathcal{U}\right\|_{F} \leq\left\|\mathcal{C}-\mathcal{C}_{N}\right\|_{F}
$$

This concludes to the convergence of $\left(\mathcal{C}_{N} \circ \mathcal{U}_{N}\right)_{N \geq 1}$ toward $\mathcal{C} \circ \mathcal{U}$, with respect to the norm of operators in $\ell^{2}(\mathbb{R})$. We turn to the proof of the inequality

$$
\mathcal{C}_{N}^{(T)} \circ \mathcal{U}_{N} \geq \eta_{T}\left(\mathcal{Q}_{N}^{(T)}\right)^{2}
$$

with a constant $\eta_{T}$ that does not depend on $N$.

Let $\mathcal{R}_{N}=\left(\mathcal{Q}_{N}^{(T)}\right)^{1 / 2}=\operatorname{diag}\left(e^{-\lambda_{k} T / 2}\right)_{1 \leq k \leq N}$ be the truncation of $\mathcal{Q}^{(T / 2)}$ and $\mathcal{I}_{N}$ 
the $N \times N$ identity matrix. In view of the diagonality of $\mathcal{R}_{N}$, it is easily seen that the former inequality is equivalent to the formula

$$
\left[\left(\mathcal{R}_{N}\right)^{-1} \mathcal{C}_{N}\left(\mathcal{R}_{N}\right)^{-1}\right] \circ\left[\left(\mathcal{R}_{N}\right)^{-1} \mathcal{U}_{N}\left(\mathcal{R}_{N}\right)^{-1}\right] \geq \eta_{T} \mathcal{I}_{N}
$$

According to (11), we get the following lower bound on the first matrix

$$
\left(\mathcal{R}_{N}\right)^{-1} \mathcal{C}_{N}\left(\mathcal{R}_{N}\right)^{-1} \geq \frac{1}{\mu_{(T / 2)}} \mathcal{I}_{N}
$$

For the second matrix $\left(\mathcal{R}_{N}\right)^{-1} \mathcal{U}_{N}\left(\mathcal{R}_{N}\right)^{-1}$, we only need to bound from below its diagonal coefficients

$$
e^{\lambda_{k} T} u_{k, k}=e^{\lambda_{k} T}\left\|e_{k}\right\|_{L^{2}(\omega)}^{2}, \quad \forall k \geq 1 .
$$

Calling for the spectral inequality established in [15, Proposition 14.6] (see also [16]) we deduce that: there exists a couple of positive constants $(\xi, L)$ depending on $\omega$ such that

$$
\left\|e_{k}\right\|_{L^{2}(\omega)}^{2} \geq L e^{-\xi\left(\lambda_{k}\right)^{1 / 2}}, \quad \forall k \geq 1 .
$$

This yields the following bound

$$
\inf _{k \geq 1}\left(e^{\lambda_{k} T} u_{k, k}\right) \geq L \inf _{k \geq 1} e^{\lambda_{k} T-\xi\left(\lambda_{k}\right)^{1 / 2}} \geq \gamma_{T, \omega}>0
$$

The constant $\gamma_{T, \omega}$ depends on the control sub-domain $\omega$ and is expected to decay to zero for short horizon $T$. Now, we invoke Schur's theorem [14, Theorem 5.3.4] (see also [35, Theorem 7.10]), which says that the smallest eigenvalues of the Hadamard product of two symmetric positive definite matrices is minored by the smallest eigenvalue of one of the matrices times the lowest diagonal entry of the other $\left({ }^{1}\right)$. Applying this theorem to our context provides the following lower bound

$$
\left[\left(\mathcal{R}_{N}\right)^{-1} \mathcal{C}_{N}\left(\mathcal{R}_{N}\right)^{-1}\right] \circ\left[\left(\mathcal{R}_{N}\right)^{-1} \mathcal{U}_{N}\left(\mathcal{R}_{N}\right)^{-1}\right] \geq \frac{\gamma_{T, \omega}}{\mu_{(T / 2)}} \mathcal{I}_{N}=\eta_{T} \mathcal{I}_{N}
$$

This is the desired result. The proof is complete.

\footnotetext{
${ }^{1}$ Let $\mathcal{A}$ and $\mathcal{B}$ be the two matrices and $\mu_{k}$ the eigenvalues of $\mathcal{A}$. We have

$$
\mathcal{A} \circ \mathcal{B} \geq\left(\min _{1 \leq k \leq N} \mu_{k}\right)\left(\min _{1 \leq k \leq N} b_{k, k}\right) \mathcal{I}
$$
}




\section{Conclusion}

Alternative proofs are presented for some observation estimates of diffusion equations. Observability are crucial for various inverse problems and null-controllability of parabolic boundary value problems (see $[20,36]$ ). The elaboration of these new and quite simple proofs uses algebraic tools and relies on the spectral properties of some structured matrices. Rewording the observation estimates in a matrix terms, one has to conduct a sharp analysis of some matrices relted to Pick's matrices. Then, by a variant of Remez' theorem, we highlight the spectral equivalence between these Pick matrices and symmetric Cauchy matrices. These latter play hence a central role in our investigation. Their major attractive feature consists in the closed form of their inverses which allow to derive the desired estimates. Finally, let us just indicate that we focus here on the 'standard' diffusion equations. Our approach applies as well to various models such as the anomalous diffusion involving fractional derivatives. We refer to $[28,25]$ for several problems where extension of these algebraic tools is possible.

\section{References}

[1] F. Ben Belgacem, S. M. Kaber- On the Dirichlet boundary controllability of the one-dimensional heat equation: semi-analytical calculations and illposedness degree. Inverse Problems 27, 055012 (2011).

[2] M. Bernkopf - A history of infinite matrices. Archive for History of Exact Sciences 4, 308-358 (1968).

[3] E. Borel — Leçons sur les fonctions entières. Paris, 1921.

[4] P. Borwein, T. Erdelyi- Generalizations of Muntz's theorem via a Remeztype inequality for Muntz spaces, J. of Amer. Math. Soc. , 10, 327-349 (1997).

[5] H. Brezis - Functional Analysis, Sobolev Spaces and Partial Differential Equations. Springer, 2011.

[6] A. L. Cauchy - Mémoire sur les fonctions alternées et sur les sommes alternés, in Exercices d'Analyse et de Physique Mathématique 2, 151-59, Bachelier, Paris (1841). 
[7] R. Courant, D. Hilbert - Methods of Mathematical Physics. New York, Interscience Publishers (1953).

[8] S. Dolecki- Observability for the one-dimensional heat equation. Studia Mathematica 48, 291-305 (1973).

[9] N. Dunford, J. T. Schwartz - Linear Operators: General theory. Interscience Publishers (1958).

[10] H. O. Fattorini, D. L. Russell - Exact controllability theorems for linear parabolic equation in one space dimension. Arch. Rat. Mech. Anal., 43, 272292 (1971).

[11] A. Fursikov, O. Yu. Imanuvilov - Controllability of Evolution Equations. Lecture Notes 34, Seoul National University, Korea (1996).

[12] E. Güichal - A lower bound of the norm of the control operator for the heat equation. J. Math. Anal. Appl. 110, 519-527 (1985).

[13] J. Hadamard - Etude sur les propriétés des fonctions entières et en particulier d'une fonction considérée par Riemann. Journal de mathématiques pures et appliquées 4), 171-216 (1893).

[14] R. A. Horn, C. R. Johnson - Topics in matrix analysis. Cambridge University Press (1991).

[15] D. Jerison, G. Lebeau - Nodal sets of sums of eigenfunctions. Harmonic analysis and partial differential equations (Chicago, IL, 1996), Univ. Chicago Press, Chicago, 223-239 (1999).

[16] J. Le Rousseau, G. Lebeau - On Carleman estimates for elliptic and parabolic operators. Applications to unique continuation and control of parabolic equations. ESAIM: Control, Optimisation and Calculus of Variations 18, 712-747 (2012).

[17] G. Lebeau, L. Robbiano - Contrôle exact de l'équation de la chaleur. Communications in Partial Differential Equations, 20, 336-356 (1995).

[18] G. Lebeau and E. Zuazua - Null-controllability of a system of linear thermoelasticity, Arch. Rational Mech. Anal., 141, 297-329 (1998).

[19] H. Li, Q. Lu, X. Zhang - Recent progress on Controllability/Observability for systems governed by Partial Differential Equations. J Syst Sci Complex 23, 527-545 (2010). 
[20] J.-L. Lions - Contrôlabilité exacte, perturbations et stabilisation des systèmes distribués. Masson, Collection RMA, Paris (1988).

[21] J.-L. Lions - Exact controllability, stabilization and perturbations for distributed systems. SIAM Rev., 30 :1-68 (1988).

[22] J.-L. Lions, E. Magenes - Problèmes aux limites non homogènes et applications. Vol. II, Dunod (1968).

[23] C. Meyer - Matrix analysis and applied linear algebra. SIAM, (2000).

[24] S. Micu, E. Zuazua - Regularity issues for the null-controllability of the linear 1-d heat equation. Systems and Control Letters 60, 406-413 (2011).

[25] S. Micu and E. Zuazua - On the controllability of a fractional order parabolic equation, SIAM J. Control Optim., 44, 1950-1972 (2006).

[26] S. Micu, E. Zuazua - An Introduction to the Controllability of Partial Differential Equations, ed. by T. Sari, Collection Travaux en Cours Hermannin Quelques Questions de Théorie du Contrôle, 67-150 (2005).

[27] D. L. Russel - Controllability and stabilizability theory for linear partial differential equations: Recent progress and open questions. SIAM Review 20, 639-739 (1978).

[28] L. Miller - A direct Lebeau-Robbiano strategy for the observability of heatlike semigroups, Discrete Contin. Dyn. Syst. Ser. B, 14, 1465-1485 (2010).

[29] L. Miller - Geometric bounds on the growth rate of null-controllability cost for the heat equation in small time, Journal of Differential Equations, 204 202-226 (2004).

[30] D. L. Russel - A unified Boundary Controllability Theory for Hyperbolic and Parabolic Partial Differential Equations, Studies in Applied Mathematics, 52, 189-212, (1973).

[31] S. Schechter - On the inversion of certain matrices. Mathematical Tables and Other Aids to Computation, 13, 73-77 (1959).

[32] L. Schwartz - Etudes des sommes d'exponentielles réelles. Thèse, Clermont-Ferrand 1943.

[33] T. I. Seidman - Boundary inequalities for eigenfunctions and boundary control theory. Journal of Differential Equations, 44, 452-459 (1982). 
[34] G. Tenenbaum, M. Tucsnac - On the null-controllability of diffusion equations. ESAIM COCV , 17, 1088-1100 (2011).

[35] F. Zhang - Matrix Theory, Basic Results and Techniques. Springer, New York, 1999.

[36] E. Zuazua - Controllability of Partial Differential Equations, [cel-00392196, version 1, 2009]. http://hal.archives-ouvertes.fr/docs/00/39/21/96/PDF/Zuazua.pdf. 